\title{
Bilateral extra-adrenal perirenal myelolipomas: a case report
}

\author{
Aniket Agarwal and Kavita Vani* (i)
}

\begin{abstract}
Background: Myelolipomas are mesenchymal tumors usually involving the adrenal gland. They are a rare entity with incidence ranging from 0.08 to $0.4 \%$ as per autopsy reports. Only $15 \%$ of these are in extra-adrenal locations such as pelvis, retroperitoneum, mediastinum, lungs, stomach, liver, spleen and kidneys. Very few (only 3) cases of bilateral extra-adrenal perirenal myelolipomas have been reported until now; and one such case has been presented in our case report.

Case presentation: A 45-year-old male presented with abdominal distension and bilateral lower limb edema for 2 months. Renal functions were mildly deranged. Ultrasound was suggestive of perirenal masses. CT scan of the abdomen confirmed the diagnosis of bilateral extra-adrenal, perirenal myelolipoma. The differentials of lipomatous retroperitoneal tumors were considered. Core biopsy from perirenal masses revealed adipose tissue with interspersed hematopoietic precursors.

Conclusion: Extra-adrenal myelolipoma are rare tumors that are not known to have malignant potential and may be symptomatic only due to mass effect. Diagnosis can be confirmed by imaging in most cases. Histopathology may not be able to confirm malignancy even in sarcoma if sample is not taken from the appropriate site, usually non-fatty softtissue components. This rare case is presented in order to add to literature, the varied presentations of extra-adrenal myelolipomas.
\end{abstract}

Keywords: Extra-adrenal myelolipoma, Retroperitoneal myelolipoma, Perirenal myelolipoma

\section{Background}

Myelolipomas are mesenchymal tumors which consist of mature adipose tissue admixed with hematopoietic cells [1]. This intriguing tumor most commonly occurs within the adrenal gland; however, it has occasionally been found within the pelvis, retroperitoneum, mediastinum, lungs, stomach, liver, spleen and kidneys [2, 3].

Myelolipoma is a rare entity with incidence ranging from 0.08 to $0.4 \%$ according to autopsy reports. Only $15 \%$ of these are in extra-adrenal locations. Perirenal extraadrenal myelolipomas are especially rare, with only few cases previously reported. We present a case of bilateral perirenal myelolipomas, which is even rarer [4-6].
Myelolipomas are usually identified incidentally. Asymptomatic and non-hemorrhagic myelolipomas do not require any treatment. The prognosis of the patient is generally excellent. However, large masses may cause mass effect and may undergo necrosis and hemorrhage. Resection is needed in symptomatic masses as they are not known to have malignant potential.

Distinguishing the mass from other close differentials on imaging is important. Contrast-enhanced CT (computed tomography) scan is the preferred investigation as it clearly demonstrates a well-defined space occupying lesion composed of fat-containing areas admixed with non-enhancing or mildly enhancing soft-tissue areas.

*Correspondence: kavita2vani@gmail.com

Department of Radiology, RML Hospital, Baba Kharak Singh Road, New

Delhi, Delhi 110001, India 


\section{Case presentation}

A 45-year-old diabetic male patient presented with abdominal distension, bilateral lower limb, edema, facial puffiness and decreased urine output for 2 months. Kidney function tests were mildly deranged (Urea and creatinine were 65 and $1.5 \mathrm{mg} / \mathrm{dL}$, respectively, with electrolytes in the normal rangesodium-137 mEq/L, chloride- $99 \mathrm{mmol} / \mathrm{L}$ and potassium-3.6 mEq/L). GFR (glomerular filtration rate) was $74 \mathrm{~mL} / \mathrm{min} / 1.73 \mathrm{~m}^{2}$. Ultrasound was suggestive of perirenal masses. Non-contrast and contrast-enhanced CT scan of whole abdomen was done for further evaluation. On imaging, as illustrated in Figs. 1, 2 and 3, there were large well-defined fat density masses in bilateral perirenal spaces with interspersed soft-tissue density areas. Both kidneys were encased within and compressed by the masses; however, there was no infiltration into the kidneys. Both adrenals were normal. There was no communication between the masses across the midline. No evidence of internal calcification was noted. No enhancement was seen in the perirenal masses on post-contrast scans. An incidental parapelvic cyst was seen on the right side. Imaging diagnosis of bilateral extra-adrenal perirenal myelolipoma was made. The differentials of lipomatous retroperitoneal tumors were considered. Ultrasound-guided core biopsy (Fig. 4) from perirenal masses revealed adipose tissue with interspersed hematopoietic precursors. The patient was treated symptomatically for renal failure. Surgical excision was planned as the masses were large and compressing both kidneys. Follow-up CT scan was advised after a 4-month interval; however, the patient was lost to follow-up.

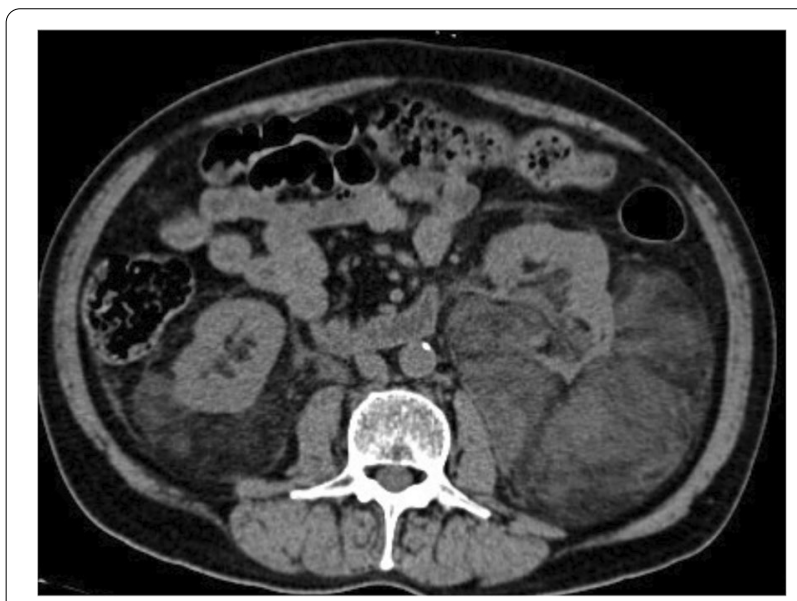

Fig. 1 Axial NCCT abdomen shows fat and interspersed soft-tissue density, bilateral perirenal masses. No calcification or hemorrhage is noted in the lesion

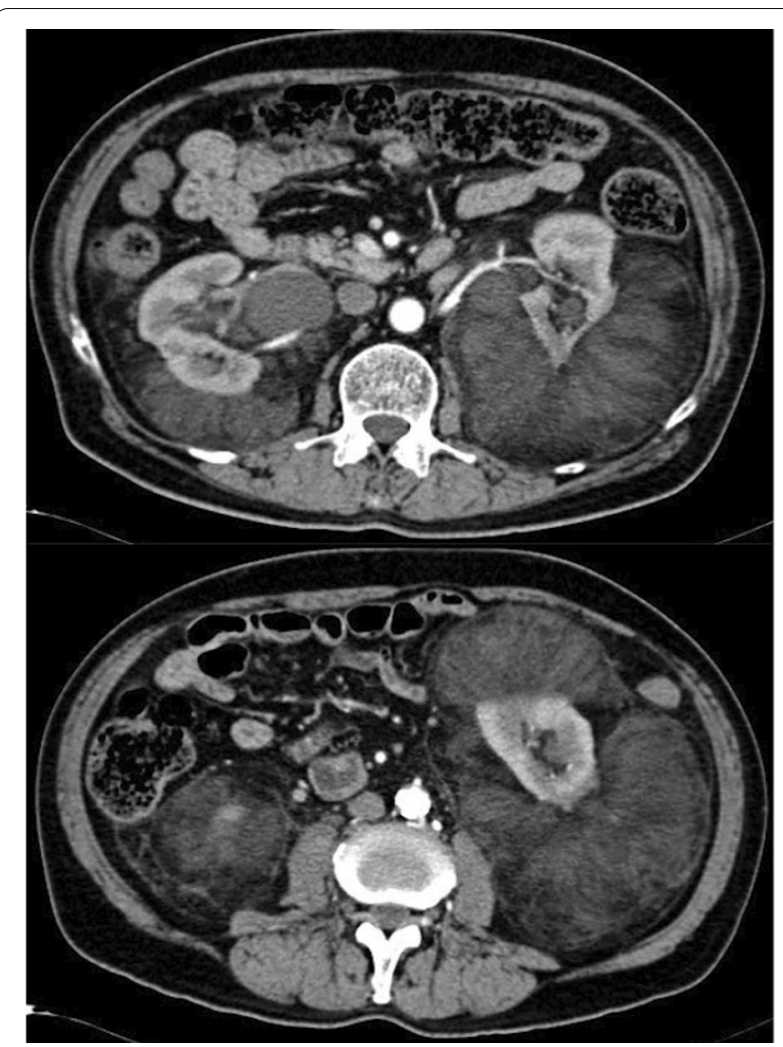

Fig. 2 Axial CECT abdomen shows normal enhancement of renal parenchyma

\section{Discussion}

Myelolipomas are benign tumors. Adrenal and extraadrenal myelolipomas are histologically similar and are composed of mature adipose cells and "trilineage" hematopoietic cells, i.e., red blood cells, white blood cells and platelets [7]. This rare tumor occurs most frequently within the adrenal gland; however, it has been occasionally found within the pelvis, retroperitoneum, mediastinum, lungs, stomach, liver, spleen and kidneys $[2,3]$.

A review of the literature shows that extra-adrenal myelolipomas exhibit a slight female predominance and are typically discovered between the ages of $50-70$ years [4]. It manifests in four different clinico-pathological patterns, i.e., isolated adrenal myelolipoma, adrenal myelolipoma with hemorrhage, extra-adrenal myelolipoma and myelolipoma associated with other adrenal disease $[6,7]$. The exact etiology is not known; however, possible hypotheses include metaplasia of adrenal cortical cells precipitated by chronic stress or degeneration, misplacement of myeloid cells during embryogenesis, or embolization of bone marrow and in response to trigger stimulus $[1,8]$.

These tumors are generally small in size and have a good prognosis. However, they may enlarge in size 


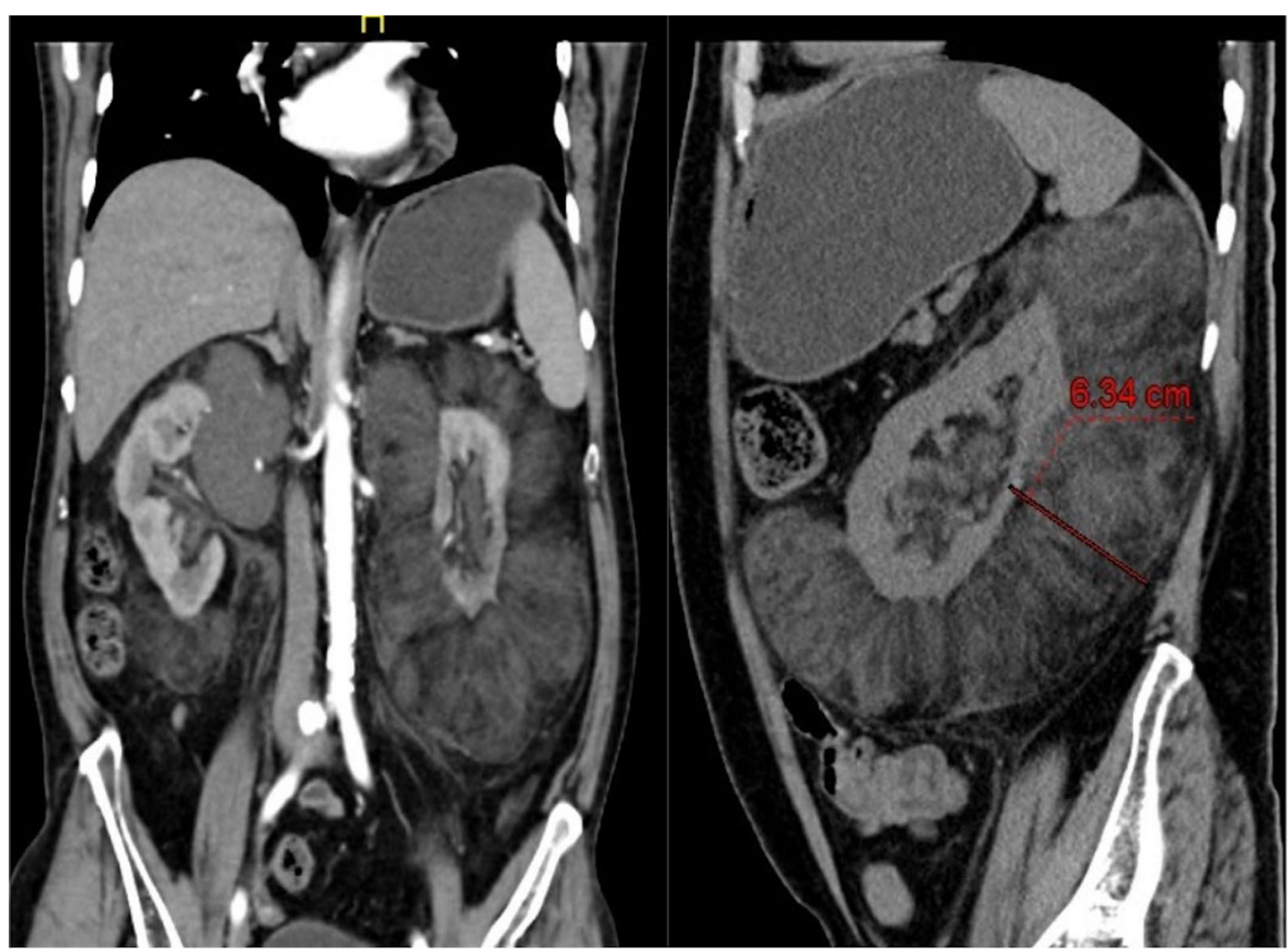

Fig. 3 Sagittal and coronal CECT abdomen shows the extent of the lesions. Bilateral perirenal masses

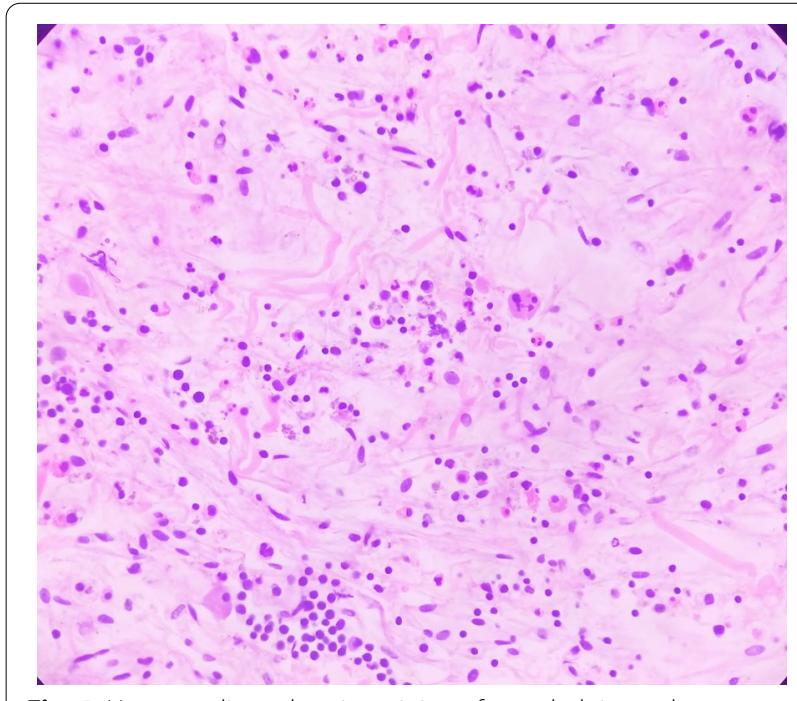

Fig. 4 Hematoxylin and eosin staining of sampled tissue shows adipose tissue with interspersed hematopoietic precursors

causing mass effect and bleeding. Sometimes, they may also be large at presentation, ranging from 5 to $19 \mathrm{~cm}$ in diameter. Malignant transformation has not yet been reported.
Adrenal myelolipomas do not pose any difficulty in diagnosis as they are heterogeneous masses composed of extracellular macroscopic fat that is easily recognized on non-contrast CT images. However, myelolipomas may pose diagnostic difficulty when present in extra-adrenal locations such as retroperitoneum, mediastinum, lungs, stomach, liver spleen, mesentery and pre-sacral regions. We present a case of bilateral perirenal extra-adrenal myelolipomas. Perirenal extra-adrenal myelolipoma is extremely rare. Only three $[6,7,9]$ cases have been reported to date.

On ultrasound, fat content appears hyperechoic. The cellular soft-tissue component appears isoechoic or hypoechoic which gives the mass a heteroechoic appearance. On non-contrast CT images, the mass appears heterogeneous with areas of fatty attenuation and interspersed soft-tissue components. It is usually well encapsulated. The mass shows no significant enhancement or mild post-contrast enhancement depending on the amount of soft-tissue component. In our case, there were large masses in bilateral perirenal spaces (left more than right), predominantly of fat attenuation, that were completely encasing and compressing both kidneys. There was associated anterior displacement of both kidneys. The lower and mid-pole cortex of the left kidney appeared to be thinned out. There was no obvious infiltration into the 
kidneys or the pararenal fascia. On post-contrast images, the mass showed no significant enhancement. The cortex of both kidneys demonstrated normal post-contrast enhancement. Bilateral adrenals were normal.

On MRI, the lesion has high signal intensity on T1WI and intermediate signal intensity on T2WI corresponding to areas of fat. Chemical shift sequences do not objectively quantify any reduction in the opposed-phase images unlike adrenal adenomas which are composed of non-adipocyte cells and are rich in intracellular fat content. However, pixels containing both fat and water may show drop in signal on opposed-phase images. MRI was not done in our case.

The differentials of a fat-containing perirenal mass are lipoma, angiomyolipoma, myelolipoma, liposarcoma and extramedullary hematopoiesis. Differentiating extraadrenal myelolipoma from well-differentiated liposarcomas, retroperitoneal myelolipoma and extramedullary hematopoiesis may be particularly difficult [6]

Lipomas are homogeneous fat-containing lesions with no or minimal soft-tissue component. Liposarcoma is the most common fat-containing retroperitoneal tumor. These are of four types: well-differentiated, pleomorphic, myxoid and round cell. The last three types are poorly differentiated and infiltrative masses. Well-differentiated liposarcomas appear as well-defined predominantly fatcontaining lesions with minimal soft-tissue attenuation and commonly contain thin septae. The appearance may be indistinguishable from lipoma, and any retroperitoneal purely fatty lesion should be considered a well-differentiated liposarcoma rather than a lipoma until proven otherwise with histologic examination [9]. However, in our case, there was no communication of the masses across the midline, that is, in the periaortic, pericaval region, and the masses were confined by anterior pararenal fascia on both sides.

Extramedullary hematopoiesis appears similar to myelolipoma on imaging as well as histology. Fine needle aspirates of both the entities show adipose tissue intermingled with hematopoietic precursors. Bone marrow biopsy may help by excluding myelofibrosis and myeloid metaplasia. A few cases of myeloid metaplasia with perirenal extramedullary hematopoiesis have been reported which appear similar to myelolipoma [10]. Moreover, extramedullary hematopoiesis is multifo$\mathrm{cal}$ and lesions are poorly circumscribed. Hence, other common sites of involvement should also be looked for in suspected cases. Skeletal system reveals features of underlying cause, such as, secondaries to bones or hematological malignancies. In our case, no abnormality was identified on the bone window. Angiomyolipomas arise from the renal cortex. Angiomyolipoma may surround the kidney but have prominent vessels within the lesion. As angiomyolipomas arise from the renal cortex, the interface between the kidney and the mass will not be smooth unlike in perirenal myelolipoma.

Core biopsy done from perirenal mass in our case revealed adipose tissue with interspersed hematopoietic precursors. The imaging diagnosis of bilateral extra-renal perirenal myelolipomas was confirmed. The patient was treated symptomatically for acute renal failure. Surgical excision was planned as the patient was symptomatic due to large masses compressing both kidneys along with a large parapelvic cyst. Follow-up $\mathrm{CT}$ scan was advised after a 4-month interval.

\section{Conclusion}

Extra-adrenal myelolipoma are rare tumors that are not known to have malignant potential and maybe symptomatic only due to mass effect or hemorrhage. Diagnosis can be confirmed by imaging in most cases. Histopathology may not be able to confirm malignancy even in sarcoma if sample is not taken from the appropriate site. This rare case is presented in order to add to the literature, the varied presentations of extra-adrenal myelolipoma.

\section{Abbreviations \\ CT: computed tomogram; MRI: magnetic resonance imaging; T1Wl: T1-weighted imaging; T2Wl:T2-weighted imaging. \\ Acknowledgements \\ None. \\ Authors' contributions \\ AA analyzed and interpreted the patient data regarding the radiological find- ings, confirmed by KV. AA was a major contributor in writing the manuscript. Both authors read and approved the final manuscript. Both authors made substantial contributions to the acquisition, analysis and interpretation of data. AA drafted the work. KV substantively revised it. Both approved the submitted version and have agreed both to be personally accountable for their own contributions and to ensure that questions related to the accuracy or integrity of any part of the work are appropriately investigated, resolved and the resolu- tion documented in the literature. All authors have read and approved the manuscript.}

\section{Funding}

Not applicable.

\section{Availability of data and materials}

Not applicable.

Ethics approval and consent to participate

Our institution does not require ethical approval for this case report.

\section{Consent for publication}

Written informed consent was obtained from the patient for publication of this case report and accompanying images.

Competing interests

The authors declare that they have no competing interests. 
Received: 19 May 2020 Accepted: 1 February 2021

Published online: 15 February 2021

\section{References}

1. Plaut A (1958) Myelolipoma in the adrenal cortex. Am J Pathol 34:487-515

2. Dieckmann KP, Hamm B, Pickartz H et al (1987) Adrenal myelolipoma: clinical, radiologic, and histologic features. Urology 29:1-8

3. Stein SH, Latour F, Frost SS (1986) Myelolipoma arising from ectopic adrenal cortex: case report and review of the literature. Am J Gastroenterol 81:999-1001

4. Ghaouti M, Znati K, Jahid A, Zouaidia F, Bernoussi Z, Mahassini N (2013) Renal myelolipoma: a rare extra-adrenal tumor in a rare site: a case report and review of the literature. J Med Case Rep 7:92

5. Olsson CA, Krane RJ, Klugo RC, Selikowitz SM (1973) Adrenal myelolipoma. Surgery 73:665-670
6. Kumar M, Deurinckx AJ (2004) Bilateral extra-adrenal perirenal myelolipoma: an imaging challenge. AJR 183:833-836

7. Temizoz O, Genchellac H, Demir MK, Unlu E, Ozdemir H (2010) Bilateral extra-adrenal myelolipomas: CT features. Br J Radiol 83(994):198-199

8. Amin MB, Tickoo SK, Schultz D (1999) Myelolipoma of the renal sinus: an unusual site for a rare extra-adrenal lesion. Arch Pathol Lab Med 123(7):631-634

9. Shaaban AM, Rezvani M, Tubay M, Elsayes KM, Woodward PJ, Menias CO (2016) Fat-containing retroperitoneal lesions: imaging characteristics, localisation, and differential diagnosis. Radiographics 36(3):710-734

10 Rapezzi D, Racchi O, Ferraris AM (1997) Perirenalextramedullaryhematopoiesis in angiogenic myeloid metaplasia: MR imaging findings. AJR 168:1388-1389

\section{Publisher's Note}

Springer Nature remains neutral with regard to jurisdictional claims in published maps and institutional affiliations.

\section{Submit your manuscript to a SpringerOpen ${ }^{\circ}$ journal and benefit from:}

- Convenient online submission

- Rigorous peer review

- Open access: articles freely available online

- High visibility within the field

- Retaining the copyright to your article

Submit your next manuscript at $\gg$ springeropen.com 